\title{
Risk Factors for Barrett's Oesophagus
}

\author{
Neel Sharma Khek Yu Ho \\ Asia Pacific Barrett's Consortium, National University Hospital Singapore, Singapore
}

\section{Key Words}

Barrett's oesophagus · Oesophageal adenocarcinoma $\cdot$ Risk factors

\begin{abstract}
Background: Barrett's oesophagus (BO) is a premalignant condition associated with the development of oesophageal adenocarcinoma (OAC). Globally, the incidence of OAC is rising. Furthermore, the prognosis regarding the morbidity and mortality of OAC is bleak, with an estimated 5 -year survival of $10-15 \%$. Hence, detection of the premalignant phase is paramount. Endoscopy and biopsy sampling is the mainstay of diagnosis. Patients may present with symptoms of gastro-oesophageal reflux disease (GORD) or be completely asymptomatic. Therefore, symptomatology alone is a poor indicator of this condition. Summary: This review highlights the current risk factors associated with the development of BO. Key Message: Primary risk factors for $\mathrm{BO}$ include male gender, increased age, a family history of the disease, long-standing GORD, smoking, obesity (specifically determined by the waist-to-hip ratio as opposed to BMI), and Caucasian race. Alcohol consumption and Helicobacter pylori are not associated with the condition. Practical Implications: By ensuring an appropriate understanding of the risk factors, clinicians can discern at-risk patients for endoscopic diagnosis and surveillance.

(C) 2016 S. Karger AG, Basel
\end{abstract}

\section{Background}

Barrett's oesophagus (BO) is a premalignant condition associated with the development of oesophageal adenocarcinoma (OAC). Endoscopy and biopsy sampling is the mainstay of diagnosis. Patients may present with symptoms of gastro-oesophageal reflux disease (GORD) or be completely asymptomatic. Therefore, symptomatology alone is a poor indicator of this condition. The evidence highlights an array of risk factors associated with the development of BO which clinicians must be aware of in order to better discern 'at-risk' patients. This review discusses the evidence in relation to such factors. 


\section{Gender and Age}

Male gender and increased age are regarded as notable risk factors. Cook et al. [1] undertook a meta-analysis and noted an overall pooled male:female sex ratio of 1.96:1 [95\% confidence interval (CI): 1.77:1, 2.17:1]. For erosive reflux, the pooled male:female sex ratio was 1.57:1 (95\% CI: 1.40:1, 1.76:1), and for non-erosive reflux disease it was 0.72:1 (95\% CI: $0.62: 1,0.84: 1)$.

Edelstein et al. [2] noted that the risk of BO increased with increased age [adjusted odds ratio (aOR) per decade for specialised intestinal metaplasia $=1.3,95 \% \mathrm{CI}$ : 1.1-1.5; visible columnar epithelium aOR $=1.4,95 \% \mathrm{CI}$ : $1.1-1.6$; long-segment columnar epithelium aOR = 1.5, 95\% CI: 1.2-1.9] and male gender (specialised intestinal metaplasia aOR $=1.5,95 \% \mathrm{CI}$ : 1.1-2.2; visible columnar epithelium aOR $=2.7,95 \% \mathrm{CI}$ : 1.6-4.5; long-segment columnar epithelium aOR $=3.9,95 \% \mathrm{CI}$ : 1.9-8.1). Gerson et al. [3] also noted male gender as a significant predictor (at the 0.05 level).

\section{Family History and Genetic Associations}

With regard to family history, one study noted that 71 of 411 probands $(17.3 \%)$ indicated an affected 1st- and/or 2nd-degree relative. Familial BO (FBO) was determined in 30 probands (7.3\%), comprising 17 of $276(6.2 \%)$ with BO, 11 of 116 (9.5\%) with OAC, and 2 of 21 (9.5\%) with adenocarcinoma of the gastro-oesophageal junction [4].

Further evidence in this regard has shown that the presence of a positive family history (1st- or 2nd-degree relative with BO, OAC, or oesophagogastric junction adenocarcinoma) was greater among case subjects than among controls (24 vs. $5 \% ; \mathrm{p}<0.005)$. Case subjects were more likely to be older ( $\mathrm{p}<0.001$ ) and male (74 vs. $43 \%$ male; $\mathrm{p}<0.0005$ ) [5]. Juhasz et al. [6] also noted a significantly higher than expected prevalence of BO in 1st-degree relatives of patients with OAC/high-grade dysplasia.

An analysis of family histories demonstrated that compared to probands with presumed 'isolated' disease, Barrett's epithelium (OAC, BO, or short-segment BO) was noted more frequently in siblings and offspring of FBO probands ( $p \leq 0.05)$. Endoscopic assessment of relatives of FBO probands demonstrated a multigeneration multiplex FBO pedigree in keeping with an autosomal dominant inherited trait [7].

An assessment of risk alleles/mutated genes associated with B0/OAC identified three major genes (MSR1, ASCC1, and CTHRC1; all $\mathrm{p}<0.001)$. In addition, 13 patients $(11.2 \%)$ with BO/OAC carried germ line mutations in MSR1, ASCC1, or CTHRC1 [8]. Ek et al. [9] estimated a statistically significant genetic variance explaining BO (array heritability $\mathrm{h}^{2} \mathrm{~g}=35 \%$; onesided $\left.\mathrm{p}=1 \times 10^{-9}\right)$ and $\mathrm{OAC}\left(\mathrm{h}^{2} \mathrm{~g}=25 \%\right.$; one-sided $\left.\mathrm{p}=2 \times 10^{-7}\right)$. The genetic correlation between BO and OAC was found to be elevated (genetic correlation $r_{g}=1.0$ ).

Su et al. [10] conducted the first genome-wide association study on BO, comprising 1,852 UK cases and 5,172 UK controls in the discovery stage and 5,986 cases and 12,825 controls in the replication stage. Variants at two loci were associated with disease risk: chromosome $6 \mathrm{p} 21$, rs9257809 $\left(\mathrm{p}_{\text {combined }}=4.09 \times 10^{-9} ;\right.$ OR $\left.=1.21\right)$, within the major histocompatibility complex locus, and chromosome 16q24, rs9936833 ( $\mathrm{p}_{\text {combined }}=2.74 \times 10^{-10} ; \mathrm{OR}=1.14$ ), for which the closest gene was FOXF1, associated with oesophageal development.

In another genome-wide association study, Levine et al. [11] compared OAC cases ( $\mathrm{n}=$ $2,390)$ and individuals with precancerous BO (n = 3,175) with 10,120 controls. For the combined case group, they noted three new associations: (1) 19p13 (rs10419226; p = $3.6 \times$ $10^{-10}$ ) in CRTC1 (encoding CREB-regulated transcription coactivator), whose aberrant activation has been associated with oncogenic activity; (2) 9q22 (rs11789015; $p=1.0 \times 10^{-9}$ ) in 
$B A R X 1$, which encodes a transcription factor involved in oesophageal specification, and (3) 3 p14 (rs2687201; $\left.\mathrm{p}=5.5 \times 10^{-9}\right)$ near the transcription factor FOXP1, which is responsible for oesophageal development.

\section{Gastro-Oesophageal Reflux Disease}

Long-standing GORD is a primary risk factor for the development of BO. Lagergren et al. [12] concluded that in patients with recurrent symptoms of reflux, as compared to those without, the ORs were 7.7 for OAC and 2.0 for adenocarcinoma of the cardia. The risk was noted to be higher for the more frequent, more severe, and longer-lasting reflux symptoms.

Lieberman et al. [13] found that 701 of 2,641 patients undergoing elective endoscopy (27\%) had GORD symptoms, and 77 of these patients had probable BO. Thrift et al. [14] analysed 683 Veterans Affairs patients undergoing elective oesophagogastroduodenoscopy, or oesophagogastroduodenoscopy concurrently with colonoscopy, in a primary care setting. Patients less than 30 years of age with frequent GORD symptoms were associated with the highest risk of $\mathrm{BO}(\mathrm{OR}=15.1)$, and the risk was noted to rise with younger age at onset of symptoms $(\mathrm{p}=0.001)$.

Rubenstein et al. [15] observed that the incidence of OAC in older men with weekly GORD symptoms was considerable (at the age of 70 years, the incidence was 60.8/100,000 personyears). The incidence of OAC in women with GORD was deemed extremely low.

\section{Smoking and Alcohol}

With regard to smoking, Andrici et al. [16] undertook a meta-analysis comprising 7,069 BO patients. The results showed that ever having smoked was associated with an increased risk of BO when compared with non-GORD controls (OR $=1.44 ; 95 \% \mathrm{CI}: 1.20-1.74$ ) or population-based controls (OR $=1.42$; 95\% CI: $1.15-1.76)$ but not GORD controls (OR $=1.18 ; 95 \%$ CI: 0.75-1.86).

Anderson et al. [17] collated information related to alcohol consumption from 230 reflux oesophagitis, $224 \mathrm{BO}$, and $227 \mathrm{OAC}$ patients and 260 frequency-matched population controls. Their results demonstrated that wine consumption was inversely associated with reflux oesophagitis (OR $=0.45 ; 95 \% \mathrm{CI}: 0.27-0.75$ ). Total alcohol consumption at 21 years of age was significantly associated with reflux oesophagitis (OR $=2.24 ; 95 \% \mathrm{CI}: 1.35-3.74)$ but not with BO or OAC (BO: OR = 1.06, 95\% CI: 0.63-1.79; OAC: OR = 1.27, 95\% CI: 0.77-2.10).

Further alcohol-related studies noted that total alcohol use was not significantly associated with the risk of BO, although an analysis by beverage type showed an inverse association for wine drinkers compared with non-drinkers ( $\geq 7$ drinks of wine per week vs. none: OR $=0.44 ; 95 \%$ CI: 0.20-0.99; multivariate analysis] [18].

Thrift et al. [19] also observed that alcohol consumption was not associated with an increased risk of BO. Conversely, moderate intake was associated with a lower risk $(14$ to $<28$ drinks per week: $\mathrm{OR}=0.39,95 \% \mathrm{CI}$ : 0.15-1.00).

\section{BMI and Waist-to-Hip Ratio}

It is generally accepted that obesity can predispose individuals to $\mathrm{BO}$, primarily due to the risk of hiatus hernia development and worsening GORD. Corley et al. [20] assessed 320 cases, 316 patients with GORD, and 317 controls. They noted a general association between 
BO and larger abdominal circumference (independent of BMI) compared with population controls (circumference $>80$ vs. $<80 \mathrm{~cm}$ : OR $=2.24,95 \% \mathrm{CI}: 1.21-4.15$ ).

A further study on this issue showed that a BMI $>30$, as compared to a lower BMI, was associated with a greater risk of BO (OR $=4.0 ; 95 \% \mathrm{CI}: 1.4-11.1 ; \mathrm{p}=0.008)$. The amount of visceral fat was approximately 1.5 -fold greater in cases than controls $\left(183 \mathrm{vs} .115 \mathrm{~cm}^{2} ; \mathrm{p}<\right.$ 0.0001 ), whereas there was less of a difference in the amount of subcutaneous fat (248 vs. $200 \mathrm{~cm}^{2} ; \mathrm{p}=0.03$ ) [21].

Hampel et al. [22] assessed the association of BMI with GORD symptoms. According to their meta-analysis, 6 of 7 studies found significant associations of BMI with erosive oesophagitis, 6 of 7 found significant associations with OAC, and 4 of 6 found significant associations with gastric cardia adenocarcinoma.

Thrift et al. [23] found no association between BMI, fat mass, and BO. Waist-to-hip ratio was significantly associated with increased BO risk (vs. all controls: OR/1 SD $=1.45 ; 95 \%$ CI: 1.03-2.04). Kubo et al. [24] noted that waist circumference was significantly associated with BO, even after adjustment for BMI.

\section{Race}

An analysis of race demonstrated that the annual incidence of BO was highest among non-Hispanic Whites (39/100,000 race-specific member-years; 95\% CI: 35-43), with lower rates among Hispanics (22/100,000; 95\% CI: 16-29), Asians (16/100,000; 95\% CI: 11-22), and Black individuals (6/100,000; 95\% CI: 2-12). Furthermore, the annual incidence was higher among men than women (31 vs. 17/100,000 in the year 2006; $p<0.01$ ). The incidence increased with age from 2 per 100,000 member-years for persons aged 21-30 years to 31 per 100,000 member-years for persons aged 61-70 years [25]. Further race analysis highlighted that Whites had a significantly higher prevalence of BO than Hispanics (6.1 vs. 1.7\%; $\mathrm{p}=$ 0.0002) and Blacks (6.1 vs. 1.6\%; $p=0.004$ ) [26].

\section{Metabolic Syndrome and Diabetes}

Research related to the metabolic syndrome showed that this condition was associated with a 2-fold increased risk of BO relative to those with (OR $=2.00 ; 95 \% \mathrm{CI}: 1.10-3.65 ; \mathrm{p}=$ 0.02 ) and those without ( $\mathrm{OR}=1.90 ; 95 \% \mathrm{CI}: 1.03-3.60 ; \mathrm{p}=0.04$ ) reflux symptoms. This association was independent of smoking, alcohol consumption, and BMI [27].

Iyer et al. [28] undertook a diabetes association study and concluded that BO cases were more likely than controls to have a higher prevalence of type 2 diabetes (5.8 vs. 5.3\%). Further analysis demonstrated that type 2 diabetes was associated with a $49 \%$ increase in the risk of BO, independent of other risk factors (OR $=1.49$; 95\% CI: 1.16-1.91).

\section{Helicobacter pylori}

Regarding Helicobacter pylori, Rubenstein et al. [29] concluded that infection with this bacterium was inversely associated with $\mathrm{BO}(\mathrm{OR}=0.53$; 95\% CI: 0.29-0.97), particularly the cagA+ strain (OR = 0.36; 95\% CI: 0.14-0.90).

Fischbach et al. [30] identified 218 cases and 439 controls, and noted that the overall OR for an association between $H$. pylori and $\mathrm{BO}$ after controlling for age and White race was 0.55 (95\% CI: 0.35-0.84). An even more pronounced inverse association was seen (OR $=0.28 ; 95 \%$ 
CI: 0.15-0.50) among participants with atrophy of the corpus or antisecretory drug use $\geq 1$ time per week.

It is generally felt that $H$. pylori lowers the risk of $\mathrm{BO}$ occurrence secondary to the development of gastritis and damaging effects on the parietal cells responsible for acid production.

\section{Conclusions}

Numerous risk factors exist for the development of BO. Such factors must be adequately determined in order to better discern at-risk patients. Furthermore, adequate patient counselling should be provided in order to reduce modifiable risks such as smoking and obesity.

\section{Disclosure Statement}

No conflicts of interest are disclosed.

\section{References}

$>1$ Cook MB, Wild CP, Forman D: A systematic review and meta-analysis of the sex ratio for Barrett's esophagus, erosive reflux disease, and nonerosive reflux disease. Am J Epidemiol 2005;162:1050-1061.

-2 Edelstein ZR, et al: Risk factors for Barrett's esophagus among patients with gastroesophageal reflux disease: a community clinic-based case-control study. Am J Gastroenterol 2009;104:834-842.

-3 Gerson LB, et al: Use of a simple symptom questionnaire to predict Barrett's esophagus in patients with symptoms of gastroesophageal reflux. Am J Gastroenterol 2001;96:2005-2012.

$\checkmark 4$ Chak A, et al: Familiality in Barrett's esophagus, adenocarcinoma of the esophagus, and adenocarcinoma of the gastroesophageal junction. Cancer Epidemiol Biomarkers Prev 2006;15:1668-1673.

$\rightarrow 5$ Chak A, et al: Familial aggregation of Barrett's oesophagus, oesophageal adenocarcinoma, and oesophagogastric junctional adenocarcinoma in Caucasian adults. Gut 2002;51:323-328.

-6 Juhasz A, et al: Prevalence of Barrett esophagus in first-degree relatives of patients with esophageal adenocarcinoma. J Clin Gastroenterol 2011;45:867-871.

7 Chak A, et al: Identification of Barrett's esophagus in relatives by endoscopic screening. Am J Gastroenterol 2004;99:2107-2114.

-8 Orloff M, et al: Germline mutations in MSR1, ASCC1, and CTHRC1 in patients with Barrett esophagus and esophageal adenocarcinoma. JAMA 2011;306:410-419.

$>9$ Ek WE, et al: Germline genetic contributions to risk for esophageal adenocarcinoma, Barrett's esophagus, and gastroesophageal reflux. J Natl Cancer Inst 2013;105:1711-1718.

$\checkmark 10$ Su Z, et al: Common variants at the MHC locus and at chromosome 16q24.1 predispose to Barrett's esophagus. Nat Genet 2012;44:1131-1136.

11 Levine DM, et al: A genome-wide association study identifies new susceptibility loci for esophageal adenocarcinoma and Barrett's esophagus. Nat Genet 2013;45:1487-1493.

12 Lagergren J, et al: Symptomatic gastroesophageal reflux as a risk factor for esophageal adenocarcinoma. N Engl J Med 1999;340:825-831.

13 Lieberman DA, Oehlke M, Helfand M: Risk factors for Barrett's esophagus in community-based practice. GORGE consortium. Gastroenterology Outcomes Research Group in Endoscopy. Am J Gastroenterol 1997;92: 1293-1297.

14 Thrift AP, et al: Age at onset of GERD symptoms predicts risk of Barrett's esophagus. Am J Gastroenterol 2013; 108:915-922.

15 Rubenstein JH, et al: Esophageal adenocarcinoma incidence in individuals with gastroesophageal reflux: synthesis and estimates from population studies. Am J Gastroenterol 2011;106:254-260.

-16 Andrici J, Cox MR, Eslick GD: Cigarette smoking and the risk of Barrett's esophagus: a systematic review and meta-analysis. J Gastroenterol Hepatol 2013;28:1258-1273.

17 Anderson LA, et al: The association between alcohol and reflux esophagitis, Barrett's esophagus, and esophageal adenocarcinoma. Gastroenterology 2009;136:799-805.

18 Kubo A, et al: Alcohol types and sociodemographic characteristics as risk factors for Barrett's esophagus. Gastroenterology 2009;136:806-815.

19 Thrift AP, et al: No significant effects of smoking or alcohol consumption on risk of Barrett's esophagus. Dig Dis Sci 2014;59:108-116. 
20 Corley DA, et al: Abdominal obesity and body mass index as risk factors for Barrett's esophagus. Gastroenterology 2007;133:34-41; quiz 311.

-21 El-Serag HB, et al: Abdominal obesity and the risk of Barrett's esophagus. Am J Gastroenterol 2005;100:21512156.

22 Hampel H, Abraham NS, El-Serag HB: Meta-analysis: obesity and the risk for gastroesophageal reflux disease and its complications. Ann Intern Med 2005;143:199-211.

-23 Thrift AP, et al: Fat mass by bioelectrical impedance analysis is not associated with increased risk of Barrett esophagus. J Clin Gastroenterol 2014;48:218-223.

24 Kubo A, et al: Sex-specific associations between body mass index, waist circumference and the risk of Barrett's oesophagus: a pooled analysis from the international BEACON consortium. Gut 2013;62:1684-1691.

25 Corley DA, et al: Race, ethnicity, sex and temporal differences in Barrett's oesophagus diagnosis: a large community-based study, 1994-2006. Gut 2009;58:182-188.

-26 Abrams JA, et al: Racial and ethnic disparities in the prevalence of Barrett's esophagus among patients who undergo upper endoscopy. Clin Gastroenterol Hepatol 2008;6:30-34.

27 Leggett CL, et al: Metabolic syndrome as a risk factor for Barrett esophagus: a population-based case-control study. Mayo Clin Proc 2013;88:157-165.

28 Iyer PG, et al: Association of Barrett's esophagus with type II diabetes mellitus: results from a large populationbased case-control study. Clin Gastroenterol Hepatol 2013;11:1108-1114.e5.

29 Rubenstein JH, et al: Association between Helicobacter pylori and Barrett's esophagus, erosive esophagitis, and gastroesophageal reflux symptoms. Clin Gastroenterol Hepatol 2014;12:239-245.

30 Fischbach LA, et al: Association between Helicobacter pylori and Barrett's esophagus: a case-control study. Am J Gastroenterol 2014;109:357-368. 